\title{
Design, Fabrication, and Characterization of a High Fill-Factor, Large Scan-Angle, Two-Axis Scanner Array Driven by a Leverage Mechanism
}

\author{
Jui-che Tsai and Ming C. Wu, Fellow, IEEE
}

\begin{abstract}
We report on the design, fabrication, and characterization of a high fill-factor, large scan-angle, two-axis scanner array. The two-axis microelectromechanical-systems (MEMS) mirror is driven by electrostatic vertical comb-drive actuators through four motion amplifying levers. The maximum mechanical rotation angles are $\pm 6.7^{\circ}$ at $75 \mathrm{~V}$ for both axes, leading to total optical scan angle of $26.8^{\circ}$. The resonant frequency is $5.9 \mathrm{kHz}$ before metallization. A linear fill factor of $98 \%$ is achieved for the one-dimensional (1-D) micromirror array. This 1D array of two-axis micromirrors was designed for $1 \times N^{2}$ wavelength-selective switches (WSSs). In addition to two-axis rotation, piston motion with a stroke of $11.7 \mu \mathrm{m}$ is also attained.

[1731]
\end{abstract}

Index Terms-Comb-drive actuator, leverage mechanism, twoaxis micromirror, wavelength-division multiplexing (WDM), wavelength-selective switch (WSS).

\section{INTRODUCTION}

M ICROELECTROMECHANICAL systems (MEMS)based wavelength-selective switches (WSS) have attracted a great deal of attention as they enable management of optical networks at the wavelength level [1]-[19]. They are also the building blocks of wavelength-selective crossconnets (WSXC) [3], [5]. The use of MEMS technologies offers low optical insertion loss and crosstalk, independence of polarization and wavelength, as well as optical transparency for bit rate and data format. Generally, MEMS-based WSSs can be divided into two categories: Free-space optical MEMS systems [1]-[17] and hybrid planar lightwave circuit (PLC)-MEMS systems [18], [19]. The free-space $1 \times N$ WSSs reported to date have a port count of $N=4$. The fundamental limitation originates in the tradeoff between the wavelength channel spacing $\left(\lambda_{\text {spacing }}\right)$ and the total number of spatial input/output ports $\left(N_{\text {spatial }}\right)$, which stems from optical diffraction. It is shown that the ratio of $N_{\text {spatial }}$ to $\lambda_{\text {spacing }}$ is confined by the grating dispersion strength as well as the effective aperture of the optical system [11]. A comprehensive discussion of the WSS scaling limit was

Manuscript received December 11, 2005; revised April 21, 2006. This work was supported by DARPA/SPAWAR under Contract N66001-00-C-8088, by the National Science Council (NSC) of Taiwan under Grants NSC 94-2218-E-002-082 and NSC 95-2221-E-002-053, and by National Taiwan University, Taiwan. Subject Editor O. Solgaard.

J.-C. Tsai is with the Graduate Institute of Electro-Optical Engineering and the Department of Electrical Engineering, National Taiwan University, Taipei 10617, Taiwan (e-mail: jctsai@ @cc.ee.ntu.edu.tw).

M. C. Wu is with the Department of Electrical Engineering and Computer Sciences and Berkeley Sensor and Actuator Center (BSAC), the University of California, Berkeley, CA 94720-1774 USA.

Digital Object Identifier 10.1109/JMEMS.2006.880291 reported in [11]. Several one-axis micromirror arrays, which are the key components of $1 \times N$ WSSs, have been reported [20]-[24]. WSS with larger port count $(N \geq 10)$ is desirable for wavelength-division multiplexing (WDM) networks. Previously, we have proposed a large port-count free-space WSS by combining a two-dimensional (2-D) collimator array with a two-axis beamsteering mechanism for each wavelength [8]-[17]. We called this architecture the $1 \times N^{2}$ WSS. We have demonstrated $1 \times N^{2}$ WSSs using two different beamsteering configurations: one with two cross-scanning one-axis micromirror arrays in a $4-f$ optical system [8]-[11], and the other with a two-axis analog micromirror array [12]-[17]. The latter is more attractive since the optical system is simpler and the port count is doubled. Our previous two-axis micromirror array was based on parallel-plate-like actuation mechanism [12]-[15], which has a limited scan angle $\left( \pm 3.4^{\circ}\right.$ and $\pm 4.4^{\circ}$, respectively, for the two axes). Larger scan angles $\left(> \pm 4^{\circ}\right.$ for both axes) are needed to fully exploit the capacity of the $1 \times N^{2}$ systems.

A $1 \times 9$ WSS has been reported recently using a hybrid PLCMEMS approach. Two PLC chips, each with five input/output ports, are stacked vertically [19]. This architecture is analogous to the free-space $1 \times N^{2}$ WSS and also requires a two-axis micromirror array.

Recently, we have proposed a novel two-axis analog micromirror array with high fill-factor and large scan-angle. This is achieved by employing a leverage actuation mechanism. The preliminary results have been reported in [16] and [17]. In this paper, we describe the detailed design, fabrication, and characterization of such devices. Large mechanical rotation angles $\left( \pm 6.7^{\circ}\right.$ for both axes at $\left.75 \mathrm{~V}\right)$, high resonant frequency (5.9 $\mathrm{kHz}$ before metallization), and high fill-factor (98\%) one-dimensional (1-D) arrays are achieved experimentally. This is accomplished by actuating each mirror with four motion-amplifying levers powered by vertical comb-drive actuators. The devices are manufactured through a surface-micromachining process. Micromirrors with similar lever-like actuations have been reported before [20], [25]-[27]. However, they are either limited to one-axis rotation [20], [25], or fabricated with bulk-micromachining processing requiring multiple-wafer etching and bonding [26], [27].

\section{DEVICE Design}

Gimbaled structures have been widely used in two-axis MEMS scanners [28]. However, the gimbals occupy a significant portion of the total area and sacrifice the fill factor 


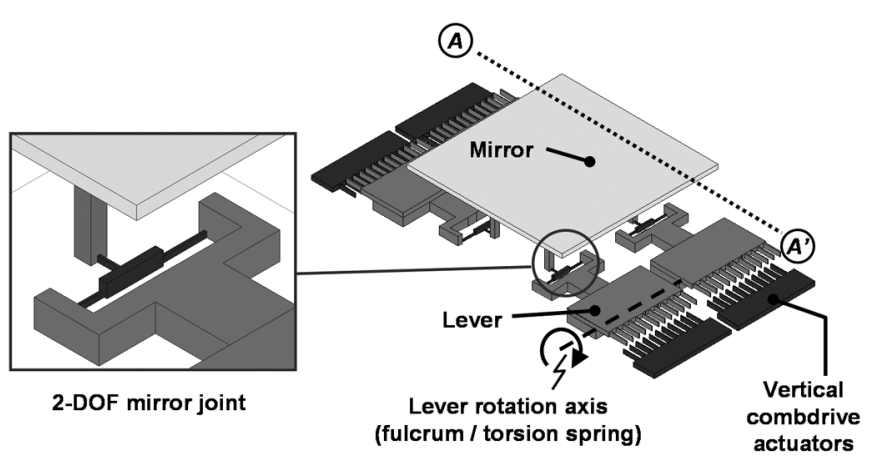

(a)

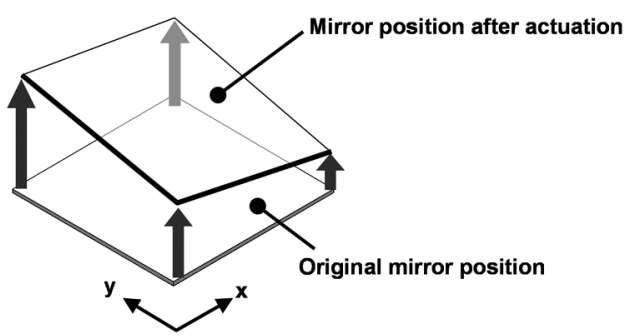

(b)

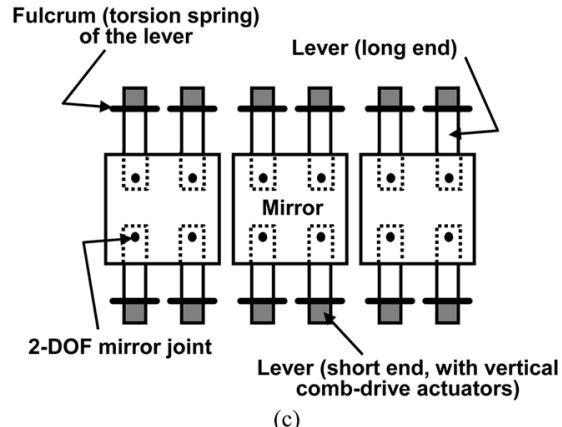

Fig. 1. (a) Schematic structure of the two-axis mirror. (b) Operation principle of the two-axis scanner. (c) Schematic of the two-axis analog micromirror array.

of the mirror array. Our previous two-axis WSS micromirror arrays employed crossbar torsion springs to eliminate gimbals and achieve a fill-factor of $>96 \%$ [12]-[15]. An electroplated two-axis scanner with a crossbar torsion spring was also previously reported for 3-D optical crossconnect applications [29]. Both of the aforementioned devices utilize parallel-plate-like electrostatic actuation for driving the mirrors. Their scan angles are limited by the pull-in effect.

Here, we employ leverage mechanism and compact compliant 2-degrees-of-freedom (DOF) joints. This leads to a gimbal-less mirror structure, which simultaneously achieves two-axis rotation, independently-controllable piston motion, and high fill-factor 1-D array. Surface-micromachining is chosen for the device fabrication and it offers excellent flexibility for high-fill factor arrays with small mirrors. The five-layer polysilicon surface-micromachining process offered by Sandia National Laboratories (Sandia Ultra-Planar, Multilevel MEMS Technology-V, or SUMMiT-V [30]) is particularly attractive for implementing such micromirrors. In this section, we describe our 2-axis WSS micromirror design that is realizable by the SUMMiT-V process. (a)

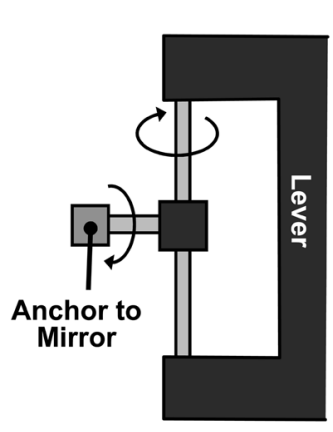

(b)

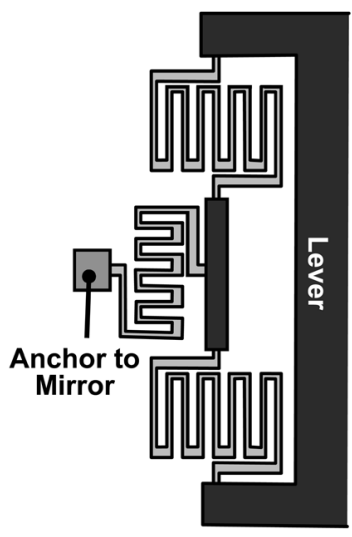

Fig. 2. (a) Stiff and (b) compliant spring designs for the 2-DOF mirror joint.

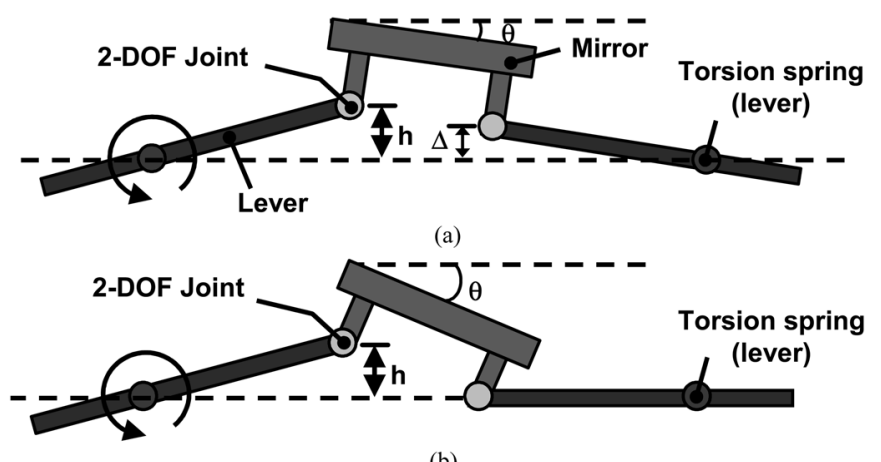

(b)

Fig. 3. Dependence of the mirror scan angle on the compliance (relative to the torsion spring constant of the lever fulcrum) of the 2-DOF joints. (a) Stiff joints. (b) Compliant joints.

\section{A. Concept and Operation Principle}

The schematic of the micromirror is shown in Fig. 1(a). Each mirror is supported by four levers. The other ends of the levers are attached to electrostatic vertical comb-drive actuators, which have been commonly used for generating large force densities in various MEMS structures [22], [31]. Similar comb-drive designs have also been adopted in our previous one-axis micromirror arrays [22]. The fulcrum (torsion spring) of the lever is positioned closer to the actuator $(30 \mu \mathrm{m}$ on the actuator side and $100 \mu \mathrm{m}$ on the mirror side) to amplify the vertical displacement at the mirror. The mirror and the lever are joined by a 2-DOF mirror joint, which translate the differential vertical displacements into 2-D tilting of the mirror. This translation is illustrated in Fig. 1(b). The four mirror corners are pushed up independently by the levers, generating the desired 2 -axis tilting through the 2-DOF joints. In addition to 2-axis tilting, this mechanism also offers piston motion. Complete two-axis scanning is then achieved by independent control of the four vertical comb-drive actuators. Fig. 1(c) is a simplified schematic showing the 1-D array formed with such two-axis scanners.

Previously, a discrete 2-D platform using a similar elevation-to-tilting mechanism was reported in [32]. However, the 


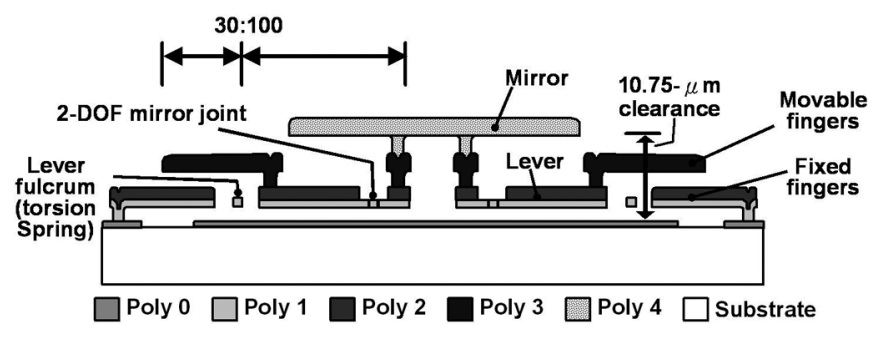

Fig. 4. Cross section of the two-axis micromirror along the $A$ - $A^{\prime}$ in Fig. 1(a).

buckling mechanism requires much larger force and also makes it more difficult to implement high fill-factor arrays.

\section{B. Design of 2-DOF Joints}

The design of the 2-DOF mirror joint plays a critical role in the performance of the two-axis scanner. As shown in the inset of Fig. 1(a), the 2-DOF mirror joint has a T-shape configuration to support rotation in two orthogonal directions. Relative to the torsion spring of the lever fulcrum, the joint can either be stiff [Fig. 2(a)] or compliant by using serpentine design as shown in Fig. 2(b). Our previous study [22] has shown that a triple-segment meander/serpentine structure possesses a torsion spring constant approximately three times smaller than that of a single-segment spring, i.e., $(3)^{1 / 2}$-time reduction in the required voltage to achieve the same rotation angle. For the work in this paper, compliant joints shown in Fig. 2(b) can produce larger scan angles, as illustrated in Fig. 3. For a stiff joint [Fig. 3(a)], when the actuated left lever produces an elevation $(h)$ at the left side of the mirror, the right side of the mirror is also lifted up by an amount $\Delta$ through mechanical coupling between the levers. This $\Delta$ reduces the mirror scan range as the tilt angle is proportional to the height difference, $h-\Delta$. On the other hand, compliant joints yield less mechanical coupling (smaller $\Delta$ ) as shown in Fig. 3(b). Therefore, larger scan angles can be achieved with compliant joints.

The four 2-DOF joints are placed symmetrically underneath the mirror, spaced by $100 \mu \mathrm{m}$. The lever amplifies the vertical displacement at the 2-DOF joint to $11.7 \mu \mathrm{m}$, leading to a maximum tilting angle of $\pm 6.7^{\circ}$ for both axes.

\section{DEVICE FABRICATION}

The cross section of the two-axis micromirror along the $A-A^{\prime}$ direction in Fig. 1(a) is shown in Fig. 4. The devices are fabricated using the SUMMiT-V process [30]. It has five polysilicon layers, including one nonreleasable ground layer (mmpoly0) and four structural layers (mmpoly1 to mmpoly4). The corresponding polysilicon layers for each structure (fixed fingers, movable fingers, levers, etc.) are labeled in Fig. 4. The mmpoly0 layer $(0.3-\mu \mathrm{m}$ thick) is designated for either the interconnecting lines or the ground planes, which shield the moving structures from the bottom dielectric. The shielding prevents any possible drift caused by the dielectric charge-up effect. The torsion springs of the lever fulcrums and 2-DOF joints are made of mmpoly1, which has a thickness of $1 \mu \mathrm{m}$. The fixed fingers of the vertical comb-drive actuators are fabricated with the laminated mmploy $1 / \mathrm{mmpoly} 2$ layer (total thickness $=2.5 \mu \mathrm{m}$ ), whereas the movable fingers are made of mmpoly $3(2.25-\mu \mathrm{m}$

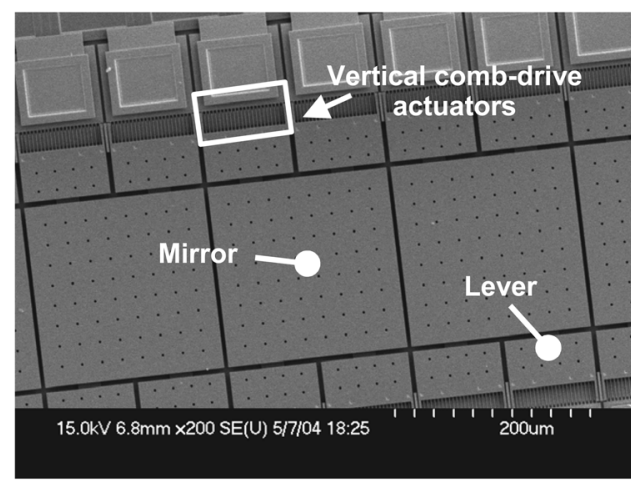

(a)

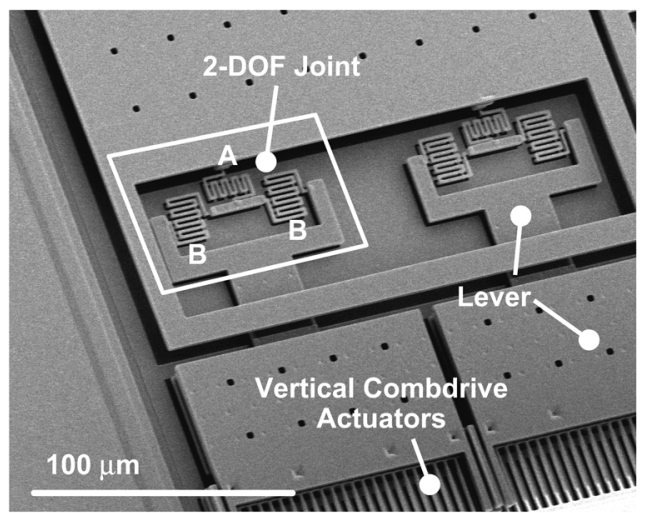

(b)

Fig. 5. SEM micrographs of the two-axis micromirrors. (a) Micromirror array. (b) Mirror partly cut to reveal the underlying levers and 2-DOF joints.

thick). The lever structure is formed by stacking mmpoly $1, \mathrm{~mm}$ poly 2 , mmpoly 3 , and mmpoly 4 to enhance the mechanical stiffness. However, underneath the mirror areas only mmpoly 1 and mmpoly 2 are used to ensure sufficient clearance $(6.25-\mu \mathrm{m})$ between the lever and the mirror. The top polysilicon layer, mmpoly4 (2.25- $\mu \mathrm{m}$ thick), is used for the mirror. The chemical-mechanical-planarization (CMP) process before the deposition of the top two polysilicon layers eliminates the topography underneath the mirrors. They also provides a large gap spacing $(10.75-\mu \mathrm{m})$ between the mirror and substrate.

Scanning electron micrographs (SEMs) of the two-axis micromirrors are shown in Fig. 5. Fig. 5(a) is part of the mirror array, while Fig. 5(b) shows the 2-DOF joints underneath the mirror. Serpentine spring A [see Fig. 5(b)] consists of nine segments, each of which has a length of $4.5 \mu \mathrm{m}$, a width of $1 \mu \mathrm{m}$, and a thickness $1 \mu \mathrm{m}$. The parameters, such as the dimensions and the number of segments, of serpentine spring B are identical with those of serpentine spring A, except that the length of each segment is $9 \mu \mathrm{m}$. The joint is 15 times more compliant than the torsion spring of the lever fulcrum, yielding $<15 \%$ mechanical coupling among the levers of the same mirror. The size of the mirror is $196 \times 196 \mu \mathrm{m}^{2}$, on a pitch of $200 \mu \mathrm{m}$. This yields a fill factor of $98 \%$. The array size is $1 \times 10$, limited by the chip area provided by the SUMMiT-V multiuser service (chip area $=3 \times 6 \mathrm{~mm}^{2}$ ).

\section{Device Characterization}

The dc characteristics of the mirror are shown in Fig. 6. They are measured using a WYKO Model: RST500, a noncontact 


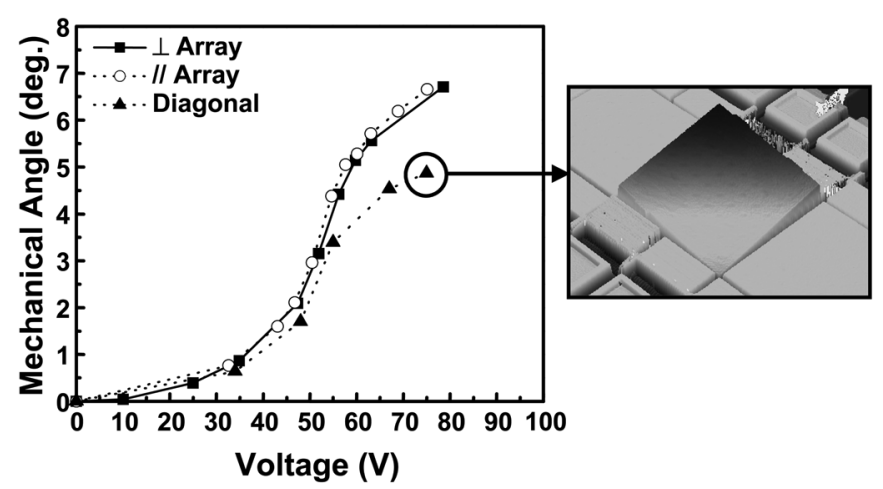

Fig. 6. DC characteristics of the two-axis mirror.

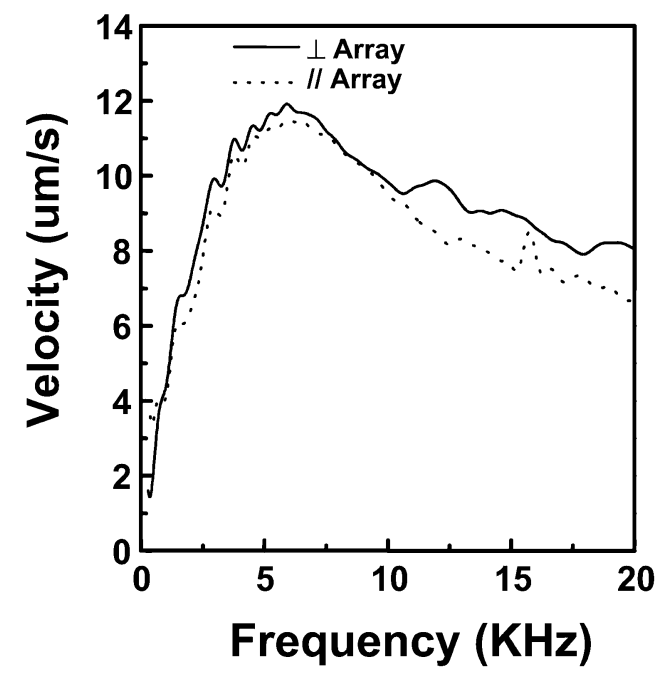

Fig. 7. Mechanical frequency response of the two-axis mirror.

white light interferometric surface profiler. The maximum mechanical scan angle is $\pm 6.7^{\circ}$ for both axes, achieved at $75 \mathrm{~V}$ bias. This provides a total optical scan angle of $26.8^{\circ}$. At these angles, the maximum vertical displacement at the 2-DOF joints is $11.7 \mu \mathrm{m}$. The scan angle in the diagonal direction is slightly smaller $\left( \pm 4.7^{\circ}\right)$ due to a longer length of the mirror base $(\sqrt{2}$ times) in the diagonal direction. For diagonal scanning, different voltages are applied on separate electrodes. The diagonal scanning curve in Fig. 6 is plotted against the highest voltage applied to the electrodes. The inset on the right is a 3-D image taken by WYKO when the mirror is diagonally tilted by $4.7^{\circ}$. The mechanical resonant frequency of the mirror is measured by a scanning laser Doppler vibrometer (manufactured by Polytec, Inc., Tustin, CA). It is $5.9 \mathrm{kHz}$ for rotation about both axes (Fig. 7). The measurement was performed before metal coating on the mirror. With 200-nm of Au and 5-nm of $\mathrm{Cr}$, the resonant frequency is estimated to be $4.5 \mathrm{kHz}$.

A prototype $1 \times N^{2}$ WSS which supports 32 output ports is built with the $1 \times 10$ array of two-axis scanners. The switching speed is characterized when the optical signal is being switched away from the input port. A step voltage of $38 \mathrm{~V}$ is applied to a pair of electrodes to scan the mirror perpendicularly to the array direction. The voltage provides a corresponding rotation angle

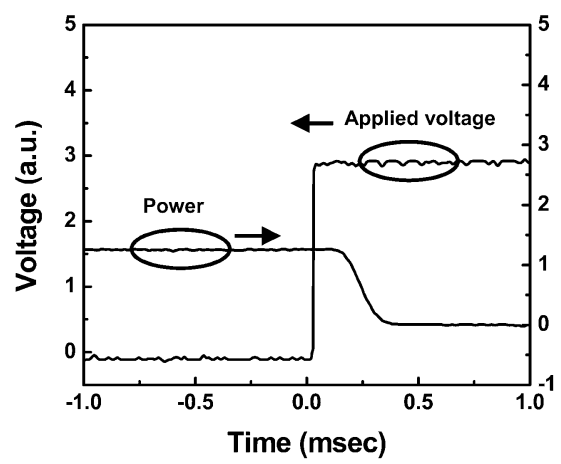

Fig. 8. Dynamic switching response of a $1 \times 32$ WSS built with the 1-D array of two-axis scanners driven by leverage mechanism.

of $1.2^{\circ}$. The switching time is measured to be less than $0.5 \mathrm{~ms}$ (Fig. 8). The detailed design and performance of the $1 \times N^{2}$ WSS have been reported elsewhere [33].

\section{CONCLUSION}

We have demonstrated a novel surface-micromachined twoaxis analog micromirror array driven by four vertical combdrive actuators through motion-amplifying levers. Such a 1-D array of two-axis scanners is the key enabling component for $1 \times N^{2}$ WSSs. The maximum mechanical scan angle is $\pm 6.7^{\circ}$ at $75 \mathrm{~V}$ for rotation about both axes. The resonant frequency is $5.9 \mathrm{kHz}$. A linear fill factor of $98 \%$ is achieved for the 1-D micromirror array. Switching time of $<0.5 \mathrm{~ms}$ has also been demonstrated in a prototype $1 \times N^{2}(1 \times 32)$ WSS. The micromirror is also capable of piston motion when all vertical comb-drive actuators are biased in unison. The maximum displacement is $11.7 \mu \mathrm{m}$.

\section{ACKNOWLEDGMENT}

The authors would like to thank L. Fan, D. Hah, M.-C. Lee, C.-H. Chi, W. Piyawattanametha, and S.-T. Hsu for technical discussions and assistance with SEM images.

\section{REFERENCES}

[1] D. M. Marom et al., "Wavelength-selective $1 \times 4$ switch for $128 \mathrm{WDM}$ channels at $50 \mathrm{GHz}$ spacing," in Proc. Optical Fiber Communication, 2002, pp. FB7-1-FB7-3, Post-deadline paper, FB7.

[2] S. Huang, J. C. Tsai, D. Hah, H. Toshiyoshi, and M. C. Wu, "Open-loop operation of MEMS WDM routers with analog micromirror array," in Proc. IEEE/LEOS Optical MEMS Conf., 2002, pp. 179-180.

[3] T. Ducellier et al., "The MWS $1 \times 4$ : A high performance wavelength switching building block," in Proc. Eur. Conf. Optical Communication, 2002, Session 2.3.1.

[4] D. M. Marom et al., "Wavelength selective $4 \times 1$ switch with high spectral efficiency, $10 \mathrm{~dB}$ dynamic equalization range and internal blocking capability," in Proc. Eur. Conf. Optical Communication, 2003, paper Mo3.5.3.

[5] D. M. Marom et al., "64 channel $4 \times 4$ wavelength-selective cross-connect for $40 \mathrm{~Gb} / \mathrm{s}$ channel rates with $10 \mathrm{~Tb} / \mathrm{s}$ throughput capacity," in Proc. Eur. Conf. Optical Communication, 2003, paper We4.P.130.

[6] J. C. Tsai, S. Huang, D. Hah, H. Toshiyoshi, and M. C. Wu, "Open-loop operation of MEMS-based $1 \times N$ wavelength-selective switch with long-term stability and repeatability," IEEE Photon. Technol. Lett., vol. 16, no. 4, pp. 1041-1043, Apr. 2004. 
[7] D. M. Marom et al., "Wavelength-selective $1 \times K$ switches using freespace optics and MEMS micromirrors: Theory, design, and implementation," IEEE/OSA J. Lightw. Technol., vol. 23, no. 4, pp. 1620-1630, Apr. 2005.

[8] J. C. Tsai, S. Huang, D. Hah, and M. C. Wu, "Wavelength-selective $1 \times N^{2}$ switches with two-dimensional input/output fiber arrays," in Proc. Conf. Lasers and Electro-Optics, 2003, CTuQ4.

[9] — " "Analog micromirror arrays with orthogonal scanning directions for wavelength-selective $1 \times N^{2}$ switches," in Proc. Transducers'03, pp. 1776-1779.

[10] $\stackrel{2}{2}, 1 \times N^{2}$ wavelength-selective switch with telescope-magnified 2D input/output fiber collimator array," in Proc. 2003 IEEE/LEOS Optical MEMS Conf., pp. 45-46.

[11] — one-axis analog micromirror arrays in a $4-f$ optical system," IEEE/OSA J. Lightw. Technol., vol. 24, no. 2, pp. 897-903, Feb. 2006.

[12] J. C. Tsai, S. Huang, and M. C. Wu, "High fill-factor two-axis analog micromirror array for $1 \times N^{2}$ wavelength-selective switches," in Proc. MEMS, 2004, pp. 101-104.

[13] J. C. Tsai and M. C. Wu, " $1 \times N^{2}$ wavelength-selective switches with high fill-factor two-axis analog micromirror arrays," in Proc. Optical Fiber Communication, 2004, paper MF42.

[14] — , " $1 \times N^{2}$ Wavelength-selective switches with tilted 2D collimator arrays for inter-channel-response suppression," in Proc. Conf. Lasers and Electro-Optics, 2004, paper CTuFF7.

[15] _ _ "Gimbal-less MEMS two-axis optical scanner array with high fill-factor," IEEE/ASME J. Microelectromech. Syst., vol. 14, no. 6, pp. 1323-1328, Dec. 2005.

[16] J. C. Tsai, L. Fan, D. Hah, and M. C. Wu, "A high fill-factor, large scanangle, two-axis analog micromirror array driven by leverage mechanism," in Proc. IEEE/LEOS Optical MEMS Conf., 2004, pp. 30-31.

[17] J. C. Tsai, L. Fan, C. H. Chi, D. Hah, and M. C. Wu, "A large port-count $1 \times 32$ wavelength-selective switch using a large scan-angle, high fillfactor, two-axis analog micromirror array," in Proc. Eur. Conf. Optical Communication, 2004, vol. 2, pp. 152-153, Paper Tu1.5.2.

[18] D. M. Marom et al., "Wavelength-selective $1 \times 2$ switch utilizing a planar lightwave circuit stack and a MEMS micromirror array," in Proc. 2004 IEEE/LEOS Optical MEMS Conf., pp. 28-29.

[19] T. Ducellier et al., "Novel high performance hybrid waveguide-MEMS $1 \times 9$ wavelength selective switch in a 32-cascade loop experiment," in Proc. Eur. Conf. Optical Communication, 2004, Th4.2.2.

[20] D. Lopez et al., "Monolithic MEMS optical switch with amplified out-of-plane angular motion," Proc. Optical MEMS, pp. 165-166, 2002.

[21] D. S. Greywall et al., "Monolithic fringe-field-activated crystalline silicon tilting-mirror devices," IEEE/ASME J. Microelectromech. Syst., vol. 12 , no. 5, pp. 702-707, Oct. 2003.

[22] D. Hah, S. T. Y. Huang, J. C. Tsai, H. Toshiyoshi, and M. C. Wu, "Low-voltage, large-scan angle MEMS analog micromirror arrays with hidden vertical comb-drive actuators," IEEE/ASME J. Microelectromech. Syst., vol. 13, no. 2, pp. 279-289, Apr. 2004.

[23] W. P. Taylor et al., "A high fill factor linear mirror array for a wavelength selective switch," J. Micromech. Microeng., vol. 14, pp. 147-152, 2004.

[24] O. Tsuboi, N. Kouma, H. Soneda, H. Okuda, X. Mi, S. Ueda, and I. Sawaki, "A high-speed comb-driven micromirror array for $1 \times N$ 80-channel wavelength selective switches," in Proc. IEEE/LEOS Optical MEMS Conf., 2004, pp. 32-33.

[25] H. Y. Lin et al., "Torsional mirror with an electrostatically driven levermechanism," Proc. Optical MEMS, pp. 113-114, 2000.

[26] V. Milanovic, G. A. Matus, and D. T. McCormick, "Tip-tilt-piston actuators for high fill-factor micromirror arrays," in Proc. Solid-State Sensor, Actuator and Microsystems Workshop, Hilton Head Island, SC, Jun. 6-10, 2004, pp. 232-237.

[27] _ - "Gimbal-less monolithic silicon actuators for tip-tilt-piston micromirror applications," IEEE J. Select. Topics Quantum Electron., vol. 10, no. 3, pp. 462-471, May/Jun. 2004.
[28] M. Whitley, J. A. Hammer, Z. Hao, B. Wingfield, and L. Nelson, "A single two-axis micromachined tilt mirror and linear array," Proc. SPIE, vol. 4985, pp. 83-94.

[29] J. H. Kim, H. K. Lee, B. I. Kim, J. W. Jeon, J. B. Yoon, and E. Yoon, "A high fill-factor micro-mirror stacked on a crossbar torsion spring for electrostatically-actuated two-axis operation in large-scale optical switch," in Proc. MEMS, 2003, pp. 259-262.

[30] [Online]. Available: http://mems.sandia.gov/tech-info/summit-v.html

[31] U. Krishnamoorthy, D. Lee, and O. Solgaard, "Self-aligned vertical electrostatic combdrives for micromirror actuation," IEEE/ASME J. Microelectromech. Syst., vol. 12, no. 4, pp. 458-464, Aug. 2003.

[32] S. L. Miller et al., Microelectromechanical apparatus for elevating and tilting a platform U. S. Patent US 6,545,385 B2.

[33] J. C. Tsai and M. C. Wu, "A high port-count wavelength-selective switch using a large scan-angle, high fill-factor, two-axis MEMS scanner array," IEEE Photon. Technol. Lett., vol. 18, no. 13, pp. 1439-1442, Jul. 2006.

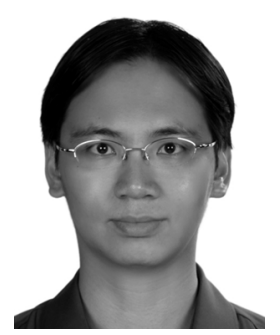

Jui-che Tsai received the B.S. degree in electrical engineering from National Taiwan University (NTU), Taiwan, in 1997. He entered the Graduate Institute of Electro-Optical Engineering at NTU after completing his undergraduate study, and received the M.S. degree in electro-optical engineering in 1999. He received the Ph.D. degree in electrical engineering from the University of California, Los Angeles (UCLA), in 2005.

Between 1999 and 2001, he served in the military as a second lieutenant. Before joining the Faculty of NTU, he was a Postdoctoral Researcher with the Department of Electrical Engineering and Computer Sciences, and Berkeley Sensor and Actuator Center (BSAC), University of California, Berkeley. He is now an Assistant Professor of the Graduate Institute of Electro-Optical Engineering and the Department of Electrical Engineering, National Taiwan University, Taiwan. His research interests include optical MEMS, optical fiber communication, and biophotonics.

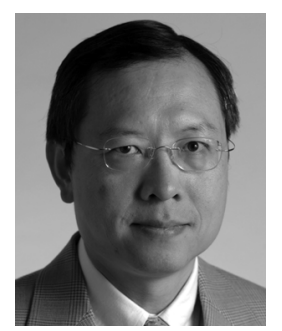

Ming C. Wu (S'82-M'83-SM'00-F'02) received the B.S. degree from National Taiwan University, and the M.S. and Ph.D. degrees from the University of California, Berkeley, in 1983, 1985, and 1988, respectively, all in electrical engineering.

Before joining the faculty of the University of California, Berkeley, he was a member of the Technical Staff at AT\&T Bell Laboratories, Murray Hill, NJ, from 1988 to 1992, and a Professor of Electrical Engineering at the University of California, Los Angeles (UCLA), from 1993 to 2004. He was also Director of the Nanoelectronics Research Facility and Vice Chair for Industrial Relations during his tenure at UCLA. In 1997, he co-founded OMM, San Diego, CA, to commercialize MEMS optical switches. He is a Professor of Electrical Engineering and Computer Sciences at the University of California, Berkeley, and Co-Director of the Berkeley Sensor and Actuator Center (BSAC). His research interests include optical MEMS (micro-electro-mechanical systems), optoelectronics, and biophotonics. He has published over 400 papers, contributed five book chapters, and holds 12 U.S. patents.

Dr. Wu is a David and Lucile Packard Foundation Fellow (1992-1997). He was the founding Co-Chair of IEEE LEOS Summer Topical Meeting on Optical MEMS (1996), the predecessor of the IEEE/LEOS International Conference on Optical MEMS. He has also served in the program committees of many technical conferences, including MEMS, OFC, CLEO, LEOS, MWP, IEDM, DRC, ISSCC; and as Guest Editor of two special issues of IEEE journals on Optical MEMS. 